\title{
Consumo de álcool entre estudantes de medicina do Sul Fluminense - RJ
}

\author{
Alcohol consumption among South Fluminense medical students - RJ
}

\section{Leonardo Silveira Gomes ${ }^{1}$, Cristina Ribeiro Dias Barroso², Vinicius Abrantes Silvestre ${ }^{3}$, Ana Cláudia do Paço Baylão ${ }^{4}$, Sônia Cardoso Moreira Garcia ${ }^{5}$, Sandro Javier Bedoya Pacheco ${ }^{6}$}

Gomes LS, Barroso CRD, Silvestre VA, Baylão ACP, Garcia SCM, Pacheco SJB. Consumo de álcool entre estudantes de medicina do Sul Fluminense - RJ / Alcohol consumption among South Fluminense medical students - RJ. Rev Med (São Paulo). 2018 maiojun.;97(3):260-6.

RESUMO: O álcool é uma das principais causas de sofrimento global, sendo uma prática comum entre estudantes universitários. Objetivo: Estimar a prevalência do consumo de álcool e a influência de gênero e ano letivo entre estudantes de Medicina de Volta Redonda, RJ. Métodos: Foi aplicado o questionário validado Teste para Identificação de Problemas Relacionados ao Álcool (AUDIT) em 265 estudantes de um curso de Medicina. Foram usadas análises de regressão logística. Resultados: A prevalência do consumo de álcool foi de $81,1 \%$. O hábito de beber no primeiro semestre $(60 \%)$ em ambos gêneros aumenta com o decorrer do curso $(p=0,012)$. A prática de beber pesado episódico (BPE) foi superior nos homens em comparação às mulheres $(p=0,01)$. Os homens apresentam chance de $130 \%$ maior de consumo nocivo de álcool em relação às mulheres $(\mathrm{p}=0,00)$. $\mathrm{O}$ grupo com consumo nocivo teve uma chance $640 \%$ maior de não cumprir as tarefas habituais e $110 \%$ maior de participar de uma agressão física $(\mathrm{p}=0,00)$. Discussão: Nossos resultados são consistentes com a literatura e mostram um notável consumo de álcool em estudantes de Medicina, sendo preocupante pela frequência e pelo volume. Conclusão: Há a necessidade de incorporação de programas de educação e prevenção do uso do álcool entre estudantes do curso de Medicina.

Descritores: Estudantes de medicina; Alcoolismo; Estilo de vida.
ABSTRACT: Alcohol is one of the main causes of global suffering, being a common practice among college students. Objective: we aimed to estimate the prevalence of alcohol consumption and the influence of gender and school year among medical students in Volta Redonda, RJ. Methods: A validated questionnaire (AUDIT) was used to identify alcohol-related problems in 265 students. Logistic regression analysis was used. Results: The prevalence of alcohol consumption was $81.1 \%$. The habit of drinking in the first semester $(60 \%)$ in both genders increased along the course $(\mathrm{p}=0.012)$. Episodic heavy drinking practice (EHD) was higher in men when compared to women (p $=0.01)$. Men present a $130 \%$ higher chance of harmful alcohol consumption than women $(\mathrm{p}=0.00)$. The group with harmful consumption had a $640 \%$ higher chance of not fulfilling the usual tasks and $110 \%$ greater of participating in a physical aggression $(\mathrm{p}=0.00)$. Discussion: Our results are consistent with the literature and show a notable consumption of alcohol in medical students, being worrying both concerning frequency and volume. Conclusion: There is need to incorporate education programs and prevention of alcohol use among medical students.

Keywords: Students, medical; Alcoholism; Life style.

O presente estudo foi aprovado pelo Comitê de Ética em Pesquisa da UniFOA, sob o parecer n ${ }^{\circ} 1.432 .034$, CAE n$^{\circ}$ 53515416.0.0000.5237. Os autores não possuem nenhum tipo de conflito de interesse.

Os autores se responsabilizam pelo conteúdo do trabalho.

1. Centro Universitário Volta Redonda/UNIFOA, Volta Redonda, RJ, BR. Estudantes de Medicina. ORCID: Gomes LS - https://orcid. org/0000-0002-0722-4766; Barroso CRD - https://orcid.org/0000-0001-8234-1148; Silvestre VA - https://orcid.org/0000-0001-83407487; Baylão ACP - https://orcid.org/0000-0001-9115-7019; E-mail: leo_silveiragomes@hotmail.com; cristinavr95@hotmail.com; abrantes_silvestre@hotmail.com; anaclaudiabaylao@gmail.com.

2. Centro Universitário Volta Redonda/UNIFOA, Volta Redonda, RJ, BR. Psicóloga, MSc. ORCID: https://orcid.org/0000-0002-50344106. E-mail: sonia.garcia@foa.org.br.

3. Fundação Oswaldo Cruz, Escola Nacional de Saúde Pública/ENSP, Departamento de Epidemiologia e Métodos Quantitativos em Saúde, Rio de Janeiro, RJ, BR. MSc, PhD. ORCID: https://orcid.org/0000-0001-6335-9940. E-mail: sandro.javier.bedoya@gmail.com. Endereço para correspondência: Cristina Ribeiro Dias Barroso. Centro Universitário de Volta Redonda (UniFOA) - Avenida Paulo Erlei Alves Abrantes, 1325. Três Poços, Volta Redonda, RJ, BR. CEP: 27240-560. Email: cristinavr95@hotmail.com 


\section{INTRODUÇÃO}

$\mathrm{O}$ consumo de álcool tem aumentado globalmente nas últimas décadas, sendo particularmente alarmante nos países de baixa e média rendas, com todos os seus efeitos sociais e consequências na saúde ${ }^{1}$. O álcool é a droga legal mais usada no Brasil, ingerida por quase $70 \%$ da população ${ }^{2}$. Ademais, o consumo de bebidas alcoólicas é uma prática comum entre estudantes universitários, sendo preocupante pela frequência e volume elevados ${ }^{3-8}$.

As características culturais, as políticas do consumo de álcool - idade permitida por lei - o curso universitário e as características de vida social dentro das faculdades podem influir substancialmente no tipo de consumo. Nos EUA, por exemplo, os maiores riscos de consumo problemático são os membros de irmandades, fraternidades ou clubes especiais de esportes ${ }^{6,9}$; em países da Europa, eles tendem a ocorrer em clubes fora do sistema educacional; já no Brasil, ocorre especialmente em festas de faculdades, onde os alunos encontram uma boa oferta de bebidas alcoólicas ${ }^{10-12}$.

Os diversos estudos já realizados ainda são insuficientes para traçar o perfil epidemiológico da população universitária brasileira em relação ao consumo de álcool. Dados como a idade de início da ingestão de bebidas alcoólicas, a diferença de consumo entre os cursos e os gêneros, a influência do ano letivo, os fatores contribuintes para o consumo elevado, o impacto no desempenho acadêmico e a sua influência nos hábitos futuros ainda se encontram sem respostas.

Sendo assim, são necessários estudos contextualizados que avaliem estas características, visando à elaboração de estratégias de prevenção bem sucedidas e duradouras. Para tanto, o presente estudo descreve a prevalência do consumo de álcool e a influência de gênero e ano letivo entre estudantes de Medicina de Volta Redonda, município da Mesorregião do Sul Fluminense.

\section{METODOLOGIA}

Foi realizado um estudo transversal, com o objetivo de estimar a prevalência do consumo de álcool e as influências do gênero e do ano letivo entre estudantes de Medicina do Centro Universitário de Volta Redonda (UniFOA). Para tanto, a pesquisa foi submetida e aprovada pelo Comitê de Ética em Pesquisa da UniFOA, sob o parecer $\mathrm{n}^{\circ}$ 1.432.034, CAE $\mathrm{n}^{\circ}$ 53515416.0.0000.5237.

A população estudada foi composta por uma amostra de 265 estudantes, distribuídos entre o primeiro e quarto ano do curso de Medicina, regularmente matriculados no ano de 2017. Os estudantes dos últimos dois anos letivos não foram considerados em virtude das dificuldades de aplicação do questionário, uma vez que a maior parte de suas atividades é realizada fora do campus universitário.

$\mathrm{O}$ instrumento de avaliação utilizado foi o Teste para Identificação de Problemas Relacionados ao Álcool
$(\mathrm{AUDIT})^{13}$, que tem como finalidade avaliar o consumo desta substância nos últimos 12 meses, identificando os indivíduos com consumo de risco, uso nocivo e dependência de álcool. A versão utilizada foi a auto aplicável, que não requer treinamento especializado, composta por dez questões ${ }^{14}$.

As três primeiras questões investigam o consumo de álcool de nível perigoso (quantidade e frequência); as três questões seguintes investigam sintomas relacionados à dependência (presença ou dependência incipiente); e as quatro questões finais referem-se ao uso prejudicial do álcool (os danos que estão sendo experimentados). $\mathrm{O}$ escore do AUDIT classifica os indivíduos por níveis de risco em Baixo Risco (zero a sete pontos), Risco Moderado (oito a 15 pontos), Uso Nocivo (16 a 19 pontos) e Provável Dependência (20 ou mais pontos), com aumento progressivo dos riscos quanto maiores forem as pontuações.

Os dados foram coletados e analisados no programa estatístico SPSS (Statistical Package for the Social Sciences - Inc. Chicago, Illinois), versão 23.0. Primeiramente, foram calculadas as prevalências do consumo de álcool em ambos os gêneros, sendo analisados: o perfil de consumo (baixo risco, risco moderado, uso nocivo, provável dependência), a prática de beber pesado episódico/BPE (consumo de seis ou mais doses de bebida alcoólica em uma ocasião), consequências diretas do consumo de álcool (agressões, não cumprimento de tarefas habituais) e percepção externa (aconselhamento de amigos, familiares ou outros). A significância estatística das medidas de associação foi realizada através do teste qui-quadrado, em nível de 5\%.

Posteriormente, foi realizada uma análise bivariada, utilizando-se o modelo de regressão logística, tendo o uso perigoso do álcool (coorte de $\geq 10$ no escore do AUDIT) como variável dependente e o ano do curso de medicina, o cumprimento das tarefas habituais, as agressões após ter bebido e a percepção externa como variáveis independentes. Foram estimados Odds Ratio (OR), Intervalo de Confiança (IC) e p-valor. Os testes foram efetuados no nível de 5\% de significância. Os dados obtidos foram organizados em tabelas cruzadas.

\section{RESULTADOS}

A amostra estudada, composta por 265 estudantes de medicina, foi predominantemente do gênero feminino (64\%). A idade variou entre 18 e 23 anos (média de 21,3 anos, DP 1.39). Quanto ao ano do curso, 87 eram do primeiro ano $(32,8 \%), 62$ do segundo $(23,4 \%), 79$ do terceiro $(29,8 \%)$ e 37 do quarto $(13,9 \%)$.

Os resultados iniciais podem ser observados na Tabela 1. A prevalência do consumo de álcool foi elevada $(81,1 \%)$ e, embora seja maior no sexo masculino, não houve diferença estatisticamente significativa entre ambos os sexos. Em relação ao perfil de consumo, embora se observe uma maior proporção de casos com baixo risco 
no gênero feminino e de risco moderado no masculino, o valor de significância se mostrou acima do estipulado como aceito. Entretanto, obtivemos um valor limítrofe que pode ter sido influenciado pelo tamanho da amostra. $\mathrm{O}$ que chama a atenção é que ambos os gêneros apresentam consequências diretas do consumo, referidas como agressões ( $10 \%$ em ambos os gêneros) e não cumprimento das tarefas habituais ( $40 \%$ entre as mulheres e $46 \%$ entre os homens), não existindo diferenças significativas. A
BPE (consumo de seis ou mais doses de bebida alcoólica em uma ocasião) foi notavelmente superior nos homens $(59 \%)$ em comparação às mulheres $(42 \%)(0,01)$. A percepção externa (aconselhamento de amigos, familiares, entre outros) aconteceu em ambos os gêneros. Na Tabela 2, pode-se observar que o hábito de beber no primeiro semestre é próximo do $60 \%$ em ambos gêneros e aumenta significativamente com o decorrer do curso de medicina $(\mathrm{p}=0,012)$.

Tabela 1. Perfil do consumo de álcool em estudantes dos três primeiros anos de Medicina, conforme o AUDIT (265 estudantes)

\begin{tabular}{|c|c|c|c|c|}
\hline \multirow[b]{2}{*}{ Variáveis } & \multirow[b]{2}{*}{ Total } & \multicolumn{3}{|c|}{ Gênero (n/\%) } \\
\hline & & Feminino & Masculino & p-valor \\
\hline \multicolumn{5}{|l|}{ Consumo de álcool ${ }^{(a)}$} \\
\hline Sim & $215(81,1)$ & $135(79,4)$ & $80(84,2)$ & \multirow[t]{2}{*}{0,33} \\
\hline Não & $50(18,9)$ & $35(20,6)$ & $15(15,8)$ & \\
\hline \multicolumn{5}{|l|}{ Perfil do consumo $^{(b)}$} \\
\hline Baixo Risco & $177(66,8)$ & $121(71,2)$ & $56(58,9)$ & \multirow[t]{4}{*}{0,06} \\
\hline Risco Moderado & $77(29,1)$ & $43(25,3)$ & $34(35,8)$ & \\
\hline Nocivo & $9(3,4)$ & $6(3,5)$ & $3(3,2)$ & \\
\hline Provável dependência & $2(0,8)$ & - & $2(2,1)$ & \\
\hline Prática do beber pesado episódico (BPE) ${ }^{(\mathrm{c})}$ & $129(48,7)$ & $73(42,9)$ & $56(58,9)$ & 0,01 \\
\hline \multicolumn{5}{|l|}{ Consequências diretas do consumo de álcool(d) } \\
\hline Agressões & $22(10,2)$ & $14(10,4)$ & $8(10,0)$ & 0,46 \\
\hline Não cumprimento das tarefas habituais & $91(42,3)$ & $54(40,0)$ & $37(46,2)$ & 0,37 \\
\hline Conselho de amigos, familiares ou outros ${ }^{(e)}$ & $24(11,2)$ & $13(9,6)$ & $11(13,7)$ & 0,09 \\
\hline
\end{tabular}

(a) $\mathrm{O}$ não consumo de álcool foi identificado quando a resposta à questão 1 do AUDIT ("Com que frequência você consome bebidas alcoólicas?) era NUNCA;

(b) Obtido a partir da pontuação gerada do AUDIT. Uso de baixo risco ou zona de risco I ( 0 a 7 pontos); uso de risco ou zona de risco II ( 8 a 15 pontos); uso nocivo ou zona de risco III (16 a 19 pontos); e provável dependência ou zona de risco IV (20 ou mais pontos);

${ }^{(c)} \mathrm{BPE}$ foi identificado quando a resposta à questão 3 do AUDIT ("Qual a frequência com que você consome seis ou mais doses de bebida alcoólica em uma ocasião?") era diferente de NUNCA;

(d) $\mathrm{O}$ incumprimento das tarefas habituais foi identificado quando à questão 5 do AUDIT ("Nos últimos 12 meses, com que frequência não conseguiu cumprir as tarefas que habitualmente lhe exigem por ter bebido?") era diferente de NUNCA. As agressões são referentes à resposta SIM, nos últimos 12 meses da questão 9 do AUDIT (“Já ficou ferido ou deixou alguém ferido alguma vez, por você ter bebido?"). A proporção é estimada a partir dos estudantes que consomem álcool;

${ }^{(e)}$ A percepção externa refere-se a um familiar, amigo, médico ou profissional de saúde que manifestou preocupação pelo seu consumo de álcool ou sugeriu que o estudante deixasse de beber nos últimos 12 meses. Valores em nível de 5\%.

Tabela 2. Hábito de beber segundo o semestre cursado na faculdade de Medicina (265 estudantes)

\begin{tabular}{cccc}
\hline \multirow{2}{*}{ Semestre cursado } & \multicolumn{3}{c}{ Habito de beber (\%) } \\
\cline { 2 - 4 } & Feminino & Masculino & Total* $^{*}$ \\
\hline $1^{0}$ & 60,7 & 61,5 & 60,8 \\
$2^{0}$ & 80,7 & 80,0 & 80,4 \\
$3^{0}$ & 84,2 & 92,8 & 87,9 \\
$4^{0}$ & 85,0 & 88,9 & 86,2 \\
$5^{0}$ & 92,9 & 94,7 & 93,6 \\
$6^{0}$ & 84,2 & 84,6 & 84,4 \\
$7^{0}$ & 76,5 & 75,0 & 76,2 \\
$8^{0}$ & 69,2 & 100,0 & 75,0 \\
\hline
\end{tabular}

*p=0,012 ao nível de $5 \%$
Após ajuste para todas as variáveis independentes analisadas, os homens apresentaram chance de $130 \%$ (OR 2,$3 ; \mathrm{IC}_{95 \%}: 1,3-4,2 ; \mathrm{p}$-valor 0,00$)$ maior de consumo nocivo de álcool em relação às mulheres (Tabela 3 ). O grupo com consumo nocivo de álcool (coorte de $\geq 10$ no escore do AUDIT) teve uma chance $640 \%$ maior de não cumprir as tarefas habituais (OR 7,4; $\mathrm{IC}_{95 \%}: 3,92-14,0 ;$ p-valor 0,00 ) e $110 \%$ maior de participar de uma agressão física (OR 12,$1 ; \mathrm{IC}_{95 \%}: 4,76-30,8$; p-valor 0,00). Da mesma forma, o grupo que teve um consumo perigoso de álcool recebeu mais conselhos de familiares, amigos e/ou médicos e/ou profissionais de saúde (OR 7,19IC ${ }_{95 \%}: 3,28-19,0$; p-valor $0,00)$. Embora visualmente observemos diferenças no uso nocivo de álcool (AUDIT >10) e o ano letivo, estas não foram estatisticamente significativas. 
Gomes LS, et al. Consumo de álcool entre estudantes de medicina do Sul Fluminense - RJ.

Tabela 3. Fatores e consequências associadas ao risco do uso nocivo de álcool (AUDIT $\geq 10$ ) em estudantes dos três primeiros anos de Medicina (265 estudantes)

\begin{tabular}{|c|c|c|c|c|c|}
\hline Características & AUDIT $\geq 10$ & AUDIT $<10$ & $\mathbf{O R}^{\mathbf{a}}$ & IC & p-valor ${ }^{b}$ \\
\hline \multicolumn{6}{|l|}{ Gênero } \\
\hline Masculino & $31(32,6)$ & $64(67,4)$ & 2,3 & $1.31-4.23$ & 0,004 \\
\hline Feminino & $29(17,1)$ & $141(82,9)$ & & & \\
\hline \multicolumn{6}{|l|}{ Ano do curso de medicina } \\
\hline Primeiro ano & $15(17.24)$ & $72(82,8)$ & 1 & - & - \\
\hline Segundo ano & $17(27,4)$ & $45(72,6)$ & 1,81 & $0.82-3.98$ & 0,139 \\
\hline Terceiro ano & $21(26,6)$ & $58(73,4)$ & 1,73 & $0.82-3.66$ & 0,147 \\
\hline Quarto ano & $7(18,9)$ & $30(81,1)$ & 1,12 & $0,41-3.02$ & 0,823 \\
\hline \multicolumn{6}{|l|}{ Tarefas habituais* } \\
\hline Não consegue cumprir & $42(46,2)$ & $49(53,8)$ & 7,4 & $3.92-14.06$ & 0,000 \\
\hline Cumpre as tarefas habituais & $18(10,3)$ & $156(89,7)$ & & & \\
\hline \multicolumn{6}{|l|}{ Agressões após ter bebido* } \\
\hline Ficou ferido ou ficou alguém ferido & $18(72,0)$ & $7(28,0)$ & 12,1 & $4,76-30,85$ & 0,000 \\
\hline Não aconteceu & $42(17,5)$ & $198(82,5)$ & & & \\
\hline \multicolumn{6}{|c|}{ Aconselhamento de amigos, familiares ou outros* } \\
\hline Recebeu conselhos*** & $16(64,0)$ & $9(36,0)$ & 7,9 & $3.28-19.08$ & 0,000 \\
\hline Não recebeu conselhos & $44(18,3)$ & $196(81,7)$ & & & \\
\hline
\end{tabular}

*Todas as variáveis são referentes aos 12 últimos meses. **Modelo de regressão logístico para análise bivariada. **Valores em nível de 5\%. ***Na categoria outros se refere a médico ou profissional de saúde.

\section{DISCUSSÃO}

A partir do presente estudo, foi verificado que cerca de $80 \%$ dos estudantes do curso universitário de Medicina consomem bebidas alcoólicas pelo menos uma vez por mês. Esta observação está de acordo com a maioria dos estudos de diferentes estados brasileiros realizados com estudantes universitários da área da saúde ${ }^{12,15-24}$. Este consumo, embora ligeiramente maior no sexo masculino, não apresentou uma diferença estatisticamente significativa em relação ao sexo feminino.

Embora não tenhamos dados pré-universitários de consumo de álcool, é possível que corresponda ao comportamento da sociedade. Há muitas décadas que a juventude brasileira vem cultivando o hábito de consumir álcool socialmente ${ }^{2,8,25}$. Estudos epidemiológicos têm apontado para o consumo elevado de álcool entre estudantes de escolas de ensino médio, alertando para a precocidade do início do uso de álcool entre adolescentes brasileiros. Geralmente, na adolescência, ocorre o primeiro contato com bebidas alcoólicas, motivado principalmente pela tendência de transgredir e de vivenciar novas experiências, como parte da construção da identidade ${ }^{26-30}$.

Os resultados mostram que os recém-ingressados no curso de Medicina apresentam uma elevada prevalência do consumo de álcool, próximo de $60 \%$ em ambos gêneros, aumentando progressivamente nos semestres posteriores $(\mathrm{p}=0,012)$. Isto permite deduzir que a maioria dos alunos inicia o uso de álcool antes de iniciarem a faculdade, havendo um acréscimo progressivo das taxas de consumo no decorrer do curso de Medicina.

No entanto, podemos observar uma ligeira redução da prevalência do consumo de álcool no sétimo e oitavo semestre. Embora exista a possibilidade de que o maior conhecimento sobre os riscos e da maior exposição a eventos adversos decorrentes de tal hábito possam ter contribuído para esta diminuição, devemos admitir que, por se tratar de um estudo longitudinal, as turmas de cada semestre incluídas no estudo podem apresentar comportamentos diferentes.

Ademais, a literatura internacional mostra que a frequência e os níveis de consumo entre os estudantes universitários de graduação são superiores aos de seus colegas não universitários ${ }^{5,31,32,33,34,35}$. Em concordância, estudos populacionais no Brasil estimam prevalências inferiores aos das populações de estudantes, que mostram valores superiores a $80 \% 0^{10,17,27,28}$.

Vários fatores podem contribuir para o aumento do consumo de álcool nos estudantes universitários. Para muitos, a universidade representa uma oportunidade de 
independência, estimulando uma maior socialização e exposição a ambientes de festas onde o alcool é ofertado. Além disso, uma parte importante dos estudantes universitários é procedente de outras cidades, e esta situação de afastamento da família pode desencadear sentimentos de solidão e depressão. Finalmente, a carga excessiva e a cobrança impostas pela vida acadêmica, a competitividade e as expectativas individuais podem gerar altos níveis de estresse e ansiedade.

Neste sentido, o álcool parece formar parte do estilo de vida dos estudantes universitários, e tal costume pode ser explicado pela busca de sensações positivas, incrementando a socialização e reduzindo a tensão ${ }^{36}$. Existem estudos detalhados que buscam descobrir as principais motivações para o consumo de álcool. Estudos na Inglaterra, por exemplo, mostraram que a principal motivação está relacionada ao prazer de beber, superando as de "ansiedade/ estresse", "para aumentar a confiança", "por hábito", "por pressões sociais" e "pressões relacionadas a provas" 37,38 .

A prática do beber pesado episódico (BPE) é definida pela quantidade de cinco ou mais doses de bebidas alcoólicas em uma única ocasião, independente da frequência de consumo diário, na semana ou no mês, ou até mesmo da embriaguez. Pode ser também denominado de uso pesado (volume excessivo) para diferenciá-lo do uso frequente (periodicidade). Alguns autores definem a BPE como o consumo superior à metade dos limites semanais seguros em um único episódio ${ }^{3,39,40}$. Estes limites semanais equivalem $\mathrm{a} \leq 21$ unidades/semana para homens e $\leq 14$ para mulheres ${ }^{41}$.

Em nosso estudo encontramos que um pouco menos da metade dos alunos realiza a prática do BPE, com uma proporção significativamente maior nos estudantes do gênero masculino. Os resultados mostram que ambos os gêneros bebem numa frequência elevada, no entanto, o gênero masculino apresenta uma maior proporção de padrões de consumo excessivo. Estatísticas similares também já foram descritas em estudos com estudantes universitários da área da saúde ${ }^{22,38,42,43}$, sendo que tais informações poderiam ser usadas em intervenções em estudantes com maior risco de consequências negativas na saúde.

De forma geral, é provável que o uso pesado, independente dos motivos acima considerados, seja mantido pela ampla aceitação social. O uso frequente (periodicidade) e o uso pesado não necessariamente estão relacionados. Estudos brasileiros mostram que a maioria dos alunos que praticam o BPE frequentam eventos com oferta de bebidas alcoólicas esporadicamente, podendo ser mensais ou quinzenais ${ }^{23}$. É dizer, portanto, que reflete uma baixa periodicidade com consumo excessivo.

Usuários de álcool que apresentam consumo excessivo possuem grande risco de danos ${ }^{31,32}$, sendo maiores ainda se aliados a maior frequência. $\mathrm{O}$ elevado nível de consumo em uma única ocasião tem sido associado a baixo desempenho, doenças sexualmente transmissíveis, danos materiais, violência e consequências criminais ${ }^{3,10,31,44-47}$. Assim sendo, é necessário atenção ao uso pesado, visto que é fundamental para estabelecer o verdadeiro impacto na população de estudantes. À medida que cresce o número de bebedores de uso pesado, o impacto global na população de estudantes é maior.

Os resultados também mostram que os alunos que fazem uso perigoso do álcool tiveram maiores dificuldades em cumprir as tarefas habituais e participaram com maior frequência de situações violentas. Este mesmo grupo também recebeu mais conselhos de familiares, amigos e/ ou médicos e/ou profissionais de saúde, o que indica uma maior percepção externa do risco desse tipo de consumo. Portanto, a necessidade da incorporação de programas de intervenção de educação e prevenção do uso do álcool torna-se evidente, sendo um assunto amplamente discutido na literatura ${ }^{48-52}$.

A educação é um processo ativo que deve estimular uma atitude crítica e reflexiva, numa interação permanente com a realidade. Para que uma estratégia cumpra seu papel transformador, deve-se contextualizar o problema. Assim, a compreensão das trajetórias do consumo de álcool em termos de motivos e padrões de consumo são fundamentais para a obtenção de resultados eficientes e duradouros. Deve-se considerar o cotidiano dos estudantes e o seu universo de valores.

Por fim, uma das limitações do presente estudo foi a não inclusão dos estudantes dos dois últimos anos letivos, o que pode restringir a sua generalização. Embora existam numerosos instrumentos para avaliar o consumo de álcool, adotamos o AUDIT pela larga utilização no nosso meio e pela validade e confiabilidade, já que foi formulado, proposto e testado pela Organização Mundial da Saúde.

\section{CONCLUSÃO}

Foi verificada uma elevada prevalência do consumo de álcool entre os estudantes do curso universitário de Medicina. Os resultados mostram que a maioria dos recém-ingressados já consome álcool e tem um aumento progressivo do consumo no decorrer dos semestres. Tal situação mostra que existe um início de uso de álcool antes da faculdade, havendo um acréscimo progressivo no decorrer dos anos letivos. Mais da metade dos alunos faz BPE, com uma proporção significativamente maior nos estudantes do gênero masculino. Assim, podemos resumir que encontramos que ambos os gêneros bebem numa frequência elevada, mas o gênero masculino apresenta em maior proporção de padrões de consumo excessivo.

As limitações do presente estudo em relação ao desenho transversal e o fato de não termos incluído estudantes dos últimos anos letivos, pode comprometer a nossa generalização. Não obstante, os presentes resultados podem contribuir para futuras pesquisas, além de estimular 
a incorporação de programas de educação e prevenção do uso de álcool entre os universitários dos cursos de Medicina.

Participação dos autores no texto: Gomes $L S$, Barroso CRD, Silvestre VA, Baylão ACP - Direta e ativa, coleta de dados, análise, discussão e conclusão; Garcia SCM, Pacheco SJB - Participação: Direta e ativa - análise, discussão e conclusão.

\section{REFERÊNCIAS}

1. World Health Organization. Global status report on alcohol and health 2014. Geneva; 2014. Available from: http://apps.who. int/iris/bitstream/handle/10665/112736/9789240692763 eng. pdf;jsessionid=1359CD10F6A8A0E6EA1EF1B3D49C9A19 ?sequence $=1$

2. Galduróz JCF, Noto AR, Nappo SA, Carlini EA. Uso de drogas psicotrópicas no Brasil: pesquisa domiciliar envolvendo as 107 maiores cidades do país - 2001. Rev Latino-Am Enfermagem. 2005;13:888-95. doi: http://dx.doi.org/10.1590/S010411692005000700017.

3. Wechsler H, Davenport A, Dowdall G, Moeykens B, Castillo S. Health and behavioural consequences of binge drinking incollege: A national survey of students at 140 campuses. JAMA. 1994;272:1671-7. doi: 10.1001/ jama.1994.03520210056032.

4. Webb E, Ashton CH, Kelly P, Kamali F. An update on British medical students' lifestyles. Med Educ. 1998;32:325-31. doi: 10.1046/j.1365-2923.1998.00204.x.

5. Kypri K, Langley JD, McGee R, Saunders JB, Williams S. High Prevalence, persistent hazardous drinking among New Zealand tertiary students. Alcohol. 2002;37:457-64. doi: 10.1093/alcalc/37.5.457.

6. Ham LS, Hope DA. College students and problematic drinking: A review of the literature. Clin Psychol Rev. 2003;23:719-59. doi: 10.1016/S0272-7358(03)00071-0.

7. Bewick BM, Mulhern B, Barkham M, Trusler K, Hill A, Stiles W. Changes in undergraduate student alcohol consumption as they progress through university. BMC Public Health. 2008;8:163. doi: https://doi.org/10.1186/1471-2458-8-163.

8. Imai FI, Coelho IZ, Bastos JL. Excessive alcohol consumption, smoking, and associated factors in a representative sample of undergraduate students from the Federal University of Santa Catarina, 2012: a cross-sectional study. Epidemiol Serv Saúde. 2014;23(3):435-46. doi: http://dx.doi.org/10.5123/S167949742014000300006 .

9. Turrisi R, Mallett KA, Mastroleo NR, Larimer ME. Heavy drinking incollege students: Who is at risk and what is being done about it? J Gen Psychol. 2014;133(4):401-20. doi: 10.3200/GENP.133.4.401-420

10. Kerr-Corrêa F, Andrade AG, Bassit AZ, Boccuto NMVF. Uso de álcool e drogas por estudantes de medicina da Unesp. Rev Bras Psiquiatr. 1999;21(2):95-100. doi: http://dx.doi. org/10.1590/S1516-44461999000200005.

11.Paduani GF, Barbosa GA, Morais JCR, Pereira JCP, Almeida MF, Prado MM, et. al. Alcohol and tobacco use among medical students of the Federal University of Uberlandia. Rev Bras Educ Med. 2008;32(1):66-75. doi: http://dx.doi.org/10.1590/ S0100-55022008000100009.
12. Baumgarten LZ, Gomes VLO, Fonseca AD. Alcohol consumption among university students in the health area of Federal University of Rio Grande/RS: subsidy to the nursing. Esc Anna Nery. 2012;16 (3):530-5. doi: http://dx.doi. org/10.1590/S1414-81452012000300015.

13. Magnabosco MB, Formigoni MLOS, Ronzani TM. Avaliação dos padrões de uso de álcool em usuários de serviços de Atenção Primária à Saúde de Juiz de Fora e Rio Pomba (MG). Rev Bras Epidemiol. 2007;10(4):637-47. doi: http://dx.doi. org/10.1590/S1415-790X2007000400021.

14. Saunders JB, Aasland OG, Babor TF, de la Fuente JR, Grant M. Development of the Alcohol Use Disorders Identification Test (AUDIT): WHO collaborative project on early detection of persons with harmful alcohol consumption. II. Addiction. 1993;88:791-804. doi: 10.1111/j.1360-0443.1993.tb02093.x.

15. Chiapetti N, Serbena CA. Uso de álcool, tabaco e drogas por estudantes da área de saúde de uma universidade de Curitiba. Psicol Reflex Crit. 2007;20(2):303-13. doi: http://dx.doi. org/10.1590/S0102-79722007000200017.

16. Amorim AVC, Kikko EO, Abrantes MM, Andrade VLA. Álcool e alcoolismo: estudo de prevalência entre discentes do curso de Medicina da UNIFENAS em Belo Horizonte - Minas Gerais. Rev Med Minas Gerais. 2008;18(1):1623. Disponível em: http://rmmg.org/artigo/detalhes/557.

17. Pedrosa AAS, Camacho LAB, Passos SRL, Oliveira RVC. Consumo de álcool entre estudantes universitários. Cad Saúde Publica. 2011;27(8):1611-21. doi: http://dx.doi.org/10.1590/ S0102-311X2011000800016.

18. Rocha LA, Lopes ACF, Martelli DRB, Lima VB, MartelliJúnior $\mathrm{H}$. Alcohol use by medical students in Minas Gerais State, Brazil. Rev Bras Med. 2011;35(3):369-75. doi: http:// dx.doi.org/10.1590/S0100-55022011000300010.

19. Carneiro EB, Braga RT, Silva LFD, Nogueira MC. Fatores associados a beber pesado episódico entre estudantes de medicina. Rev Bras Educ Med. 2012;36(4):524-30. doi: http:/ dx.doi.org/10.1590/S0100-55022012000600011.

20. Barbosa FL, Barbosa RL, Barbosa MCL, Aguiar DL, Figueiredo IA, Ribeiro AC, et. al. alcohol consumption among medical students at the Federal University of Maranhão, Brazil. Rev Bras Educ Med. 2013;37(1):89-95. doi: http:// dx.doi.org/10.1590/S0100-55022013000100013.

21. Faria YO, Gandolfi L, Moura LBA. Prevalence of risk behaviors in young university students. Acta Paul Enferm. 2014;27(6):591-5. doi: http://dx.doi.org/10.1590/19820194201400096 .

22. Cardoso FM, Barbosa HA, Costa FM, Vieira MA, Caldeira AP. Fatores associados à prática do bingedrinking entre estudantes da área da saúde. Rev CEFAC. 2015;17(2):475-84. doi: http://dx.doi.org/10.1590/1982-021620158914.

23. Pelicioli M, Barelli C, Gonçalves CCB, Hahn SR, Scherer JI. Alcohol consumption and episodic heavy drinking among undergraduate students from the health area of a Brazilian university. J Bras Psiquiatr. 2017;66(3):150-6. doi: http:// dx.doi.org/10.1590/0047-2085000000164.

24. Pinheiro MA, Torres LF, Bezerra MS, Cavalcante RC, Alencar RD, Donato AC, Campêlo CPB, Gomes IP, Alencar $\mathrm{CH}$, Cavalcanti LPG. Prevalence and Associated Factors of Alcohol Consumption and Smoking among Medical Students in Northeastern Brazil. Rev Bras Educ Med. 
2017;41(2):231-50. doi: http://dx.doi.org/10.1590/1981$52712015 \mathrm{v} 41 \mathrm{n} 2 \mathrm{rb} 20160033$.

25. Muza GM, Bettiol H, Mucillo G, Babieri MA. Consumo de substâncias psicoativas por adolescentes escolares de Riberão Preto, SP (Brasil). I - Prevalência de consumo por sexo, idade e tipo de substancia. Rev Saúde Pública. 1997;31(1):21-9. doi: 10.1590/S0034-89101997000100005.

26. Moreira LB, Fuchs FD, Moraes RS, Bredemeir M, Cardoso S. Prevalência de tabagismo e fatores associados em área metropolitana da Região Sul do Brasil. Rev Saúde Pública. 1995;29(1):46-51. doi: http://dx.doi.org/10.1590/S003489101995000100008.

27. Silva EF, Pavani RAB, Moraes MS, Neto FC. Prevalência do uso de drogas entre escolares do ensino médio do Município de São José do Rio Preto, São Paulo. Brasil. Cad Saúde Pública. 2006;22(6):1151-8. doi: 10.1590/S0102$311 \times 2006000600004$.

28. Guimaraes JL, Godinho PH, Cruz R, Kappan JL, Junior LAT. Consumo de drogas psicoativas por adolescentes escolares de Assis, SP. Rev Saúde Pública. 2004;38(1):130-2. doi: 10.1590/S0034-89102004000100018.

29. Micheli D, Formigoni MLOS. Drug use by Brazilian students: associations with family, psychosocial, health, demographic and behavioral characteristic. Addiction. 2004;99:570-8. doi: 10.1111/j.1360-0443.2003.00671.x.

30. Sanceverino SL, Abreu JLC. Aspectos epidemiológicos do uso de drogas entre estudantes do ensino médio no município de Palhoça. Ciência Saúde Coletiva. 2004;9(4):1047-56. doi: http://dx.doi.org/10.1590/S1413-81232004000400011.

31. Wechsler H, Lee JE, Kuo M, Seibring M, Nelson TF, Lee H. Trends in college binge drinking during a period of increased prevention efforts. Findings from 4 Harvard School of Public Health College Alcohol Study surveys: 1993-2001. J Am Coll Health. 2002;50(5):203-17. doi: https://doi. org/10.1080/07448480209595713.

32. Wechsler H, Dowdall GW, Davenport A, Castillo S. Correlates of college student binge drinking. Am J Public Health. 1995;85(7):921-6. doi: 10.1111/j.1530-0277.2011.01681.x.

33. O'Malley PM, Johnston LD. Epidemiology of alcohol and other drug use among American college students. J Studies Alcohol. 2002;14:23-39. doi: https://doi.org/10.15288/ jsas.2002.s14.23.

34. Dawson DA, Grant BF, Stinson FS, Chou PS: Another look at heavy episodic drinking and alcohol use among college and noncollege youth. J Stud Alcohol. 2004;65:477-89. doi: https://doi.org/10.15288/jsa.2004.65.477.

35. Johnston LD, O’Malley PM, Bachman JG, Schulenberg J. Monitoring the future. national survey results on drug use, 1975-2008. Bethesda, MD: National Institute on Drug Abuse (NIDA); 2009. v.1, p.5-27. Disponível em: http://www. monitoringthefuture.org/pubs/monographs/vol1_2008.pdf.

36. Oei TPS, Morawska A. A cognitive model of binge drinking: The influence of alcohol expectancies and drinking refusal self-efficacy. Addictive Behav. 2004;29:159-79. doi: https:// doi.org/10.1016/S0306-4603(03)00076-5.

37. Newbury-Birch D, Walshaw D, Kamali F. Factors influencing alcohol and illicit drug use amongst medical students. Drug Alcohol Depend. 2000;59:125-30. doi: https://doi. org/10.1016/S0376-8716(99)00108-8.

38. Newbury-Birch D, Lowry RJ, Kamali F. The changing patterns of drinking, illicit drug use, stress, anxiety and depression in dental students in a UK dental school: a longitudinal study. Br Dent J. 2002;192(11):646-9. doi: 10.1038/sj.bdj.4801448.

39. Moore L, Smith C, Catford J. Binge drinking: prevalence, patterns and policy. Health Educ Res. 1994;9(4):497-505. doi: 10.1186/s13561-014-0040-4.

40. Newbury-Birch D, Walshaw D, Kamali F. Drink and drugs: from medical students to doctors. Drug Alcohol Depend. 2001;64(3):265-70. doi: https://doi.org/10.1016/S03768716(01)00128-4.

41. Allan DT, Christopher CH, Cook RT, John AH. The royal college of physicians report on alcohol: guidelines for managing wernicke's encephalopathy in the accident and emergency department. Alcohol Alcoholism. 2002;37(6):51321. doi: 10.1093/alcalc/37.6.513.

42. File SE, Mabbutt PS, Shaffer J. Alcohol consumption and lifestyle in medical students. J Psychopharmacol. 1994; 8:2226. DOI: doi/abs/10.1177/026988119400800104.

43. Underwood B, Fox K. A survey of alcohol and drug use among UK based dental undergraduates. Br Dent J. 2000; 18:314-7. doi: 10.3390/dj4010002.

44. Berkowitz AD, Perkins HW. Problem drinking among college students: are view of recent research. J Am Coll Health. 1986;35(1):21-28. DOI: 10.1080/07448481.1986.9938960.

45. Delk EW, Meilman PW. Alcohol use among college students in Scotland compared with norms from the United States. J Am Coll Health. 1996;44:274-81. doi: 10.1080/07448481.1996.9936855.

46. Hosier G, Cox WM. Personality and motivational correlates of alcohol consumption and alcohol-related problems among excessive drinking university students. Addictive Behav. 2011;36:87-94. doi: 10.1016/j.addbeh.2010.08.029.

47. Jones KA, Chryssanthakis A, Groom M. Impulsivity and drinking motives predict problem behaviours relating to alcohol use in University students. Addictive Behav. 2014;39:289-96. doi: 10.1016/j.addbeh.2013.10.024.

48. Pickard M, Bates L, Dorian M, Greig H, Saint D. Alcohol and drug use in second-year medical students at the University of Leeds. Med Educ. 2000;34(2):148-50. doi: 10.1046/j.13652923.2000.00491.x.

49. Wallace P. Medical students, drugs and alcohol: time for medical schools to take the issue seriously. Med Educ. 2000;34(2):86-7. DOI: http://dx.doi.org/10.1590/S010055022012000600011 .

50. Granville-Chapman JE, Yu K, White PD. A follow-up survey of alcohol consumption and knowledge in medical students. Alcohol Alcohol. 2001;36(6):540-3. doi: 10.1093/ alcalc/36.6.540.

51. Keller S, Maddock JE, Laforge RG, Velicer WF, Basler HD. Binge drinking and health behavior in medical students. Addict Behav. 2007;32(3):505-15. doi: 10.1016/j. addbeh.2006.05.017.

52. Frank E, Elon L, Naimi T, Brewer R. Alcohol consumption and alcohol counselling behaviour among US medical students: cohort study. BMJ. 2008;337(1):2155. doi: https:// doi.org/10.1136/bmj.a2155.

Submetido em: 16.02.18

Aceito em: 11.04.18 